OPEN ACCESS

Edited by:

Evelyn K. F. Yim

University of Waterloo, Canada

Reviewed by:

Lohitash Karumbaiah, University of Georgia, United States

Silviya Petrova Zustiak,

Saint Louis University, United States

*Correspondence:

Ana M. Magariños

amagarinos@rockefeller.edu

Brendan A. C. Harley

bharley@illinois.edu

Specialty section:

This article was submitted to Biomaterials,

a section of the journal

Frontiers in Materials

Received: 31 January 2018 Accepted: 08 June 2018

Published: 28 June 2018

Citation:

Magariños AM, Pedron S, Creixell M. Kilinc M, Tabansky I, Pfaff DW and Harley BAC (2018) The Feasibility of Encapsulated Embryonic Medullary

Reticular Cells to Grow and Differentiate Into Neurons in

Functionalized Gelatin-Based Hydrogels. Front. Mater. 5:40. doi: 10.3389/fmats.2018.00040

\section{The Feasibility of Encapsulated Embryonic Medullary Reticular Cells to Grow and Differentiate Into Neurons in Functionalized Gelatin-Based Hydrogels}

\author{
Ana M. Magariños ${ }^{1 *}$, Sara Pedron ${ }^{2}$, Marc Creixell ${ }^{1}$, Murat Kilinc ${ }^{1}$, Inna Tabansky ${ }^{1}$, \\ Donald W. Pfaff ${ }^{1}$ and Brendan A. C. Harley ${ }^{2,3 *}$
}

${ }^{1}$ Laboratory of Neurobiology and Behavior, The Rockefeller University, New York, NY, United States, ${ }^{2}$ Carl R. Woese Institute for Genomic Biology, University of Illinois at Urbana-Champaign, Urbana, IL, United States, ${ }^{3}$ Department of Chemical and Biomolecular Engineering, University of Illinois at Urbana-Champaign, Urbana, IL, United States

The study of the behavior of embryonic neurons in controlled in vitro conditions require methodologies that take advantage of advanced tissue engineering approaches to replicate elements of the developing brain extracellular matrix. We report here a series of experiments that explore the potential of photo-polymerized gelatin hydrogels to culture primary embryonic neurons. We employed large medullary reticular neurons whose activity is essential for brain arousal as well as a library of gelatin hydrogels that span a range of mechanical properties, inclusion of brain-mimetic hyaluronic acid, and adhesion peptides. These hydrogel platforms showed inherent capabilities to sustain neuronal viability and were permissive for neuronal differentiation, resulting in the development of neurite outgrowth under specific conditions. The maturation of embryonic medullary reticular cells took place in the absence of growth factors or other exogenous bioactive molecules. Immunocytochemistry labeling of neuron-specific tubulin confirmed the initiation of neural differentiation. Thus, this methodology provides an important validation for future studies of nerve cell growth and maintenance.

Keywords: 3D cell culture, hyaluronic acid, neurons, nucleus gigantocellularis, brain development, biomaterial models, gelatin hydrogels

\section{INTRODUCTION}

Large reticular formation neurons in the medulla, neurons in the nucleus gigantocellularis (NGC), have recently been identified as master cells responsible for the arousal of the mammalian brain (Calderon et al., 2016). NGC neurons are located just above the spinal cord and are essential for supporting generalized CNS arousal (Pfaff, 2006), responsible for facilitating the initiation and vigor of all motivated behaviors. They may have evolved from the large Mauthner cells similarly placed in the fish hindbrain and similarly responsible for the rapid initiation of behavior (Pfaff et al., 2014). These nerve cells have extremely wide dendritic arbors and are able to respond to stimuli in all sensory modalities tested (Martin et al., 2010, 2011). Their axonal distribution encompasses ascending projections to the thalamus 
and hypothalamus and descending projections to all levels of the spinal cord, bilaterally (Jones and Yang, 1985). Their increase in excitability activates the electroencephalogram in a deeply anesthetized animal (Wu et al., 2007) and their growing ability to fire trains of action potentials as a mouse pup grows from postnatal day 3 to postnatal day 6 is correlated with the increased behavioral arousal of the mouse pup during those 4 days (Liu et al., 2016). As a result, increasingly detailed studies of these neurons under controlled conditions may yield significant new information to help understand their roles in brain function and response to injury. However, such studies are difficult in traditional in vivo settings.

It is generally acknowledged that standard $2 \mathrm{D}$ in vitro systems lack important architectural features of native tissues. Tissue engineering approaches have increasingly been used to recreate a myriad of tissue microenvironments in the context of tissue regeneration or for the development of in vitro model systems for the study of biological processes, personalized medicine, and pharmacological testing (Neves et al., 2016; Pradhan et al., 2016; Caballero et al., 2017). However, fabrication of ex vivo cerebral tissue has been elusive, due to the high complexity, including multiple hierarchical levels of interconnected neuronal networks. Tumor models have been described within hydrogel matrices (Xiao et al., 2017) and neural cells have been encapsulated successfully within synthetic (Mckinnon et al., 2013), natural (Palazzolo et al., 2015; Alessandri et al., 2016), and protein (Lampe et al., 2013) hydrogels. Besides the use of bulk hydrogels, more sophisticated fabrication techniques have been used such as three-dimensional printing ( $\mathrm{Gu}$ et al., 2016), lithography (Gurkan et al., 2013), and compartmentalized scaffolds (TangSchomer et al., 2014). Organoids of certain regions of the brain have been described (Di Lullo and Kriegstein, 2017) but they lack the oxygen and nutrient diffusion provided by biocompatible hydrogel matrices. In order to study the development of these NGC neurons, complementary to in vivo studies, 3dimensional growth platforms may be an ideal framework that could mimic features of the brain's unique extracellular matrix.

Hydrogel technologies have become increasingly well developed, with recent efforts beginning to utilize a series of hydrogel platforms to study features of brain tumor such as primary glioblastoma (GBM) or tumor metastasis to the brain (Rape et al., 2014; Heffernan et al., 2015; Kingsmore et al., 2016; Chung et al., 2017; Pedron et al., 2017a,b). Recent efforts in our lab have focused on the development of a class of photopolymerizable gelatin hydrogels. Gelatin is a natural polymer that provides cell binding sites (e.g., RGD) and degradation moieties (e.g., MMP sensitive). The functionalization with methacrylamide groups allows for the formation of a covalently crosslinked gel (Pedron and Harley, 2013). Hyaluronic acid (HA) is the major component in the fetal mammalian brain ECM (Baier et al., 2007) and it has been used to culture neuronal cells (Seidlits et al., 2010). Efforts in our lab have demonstrated covalent incorporation of methacrylated hyaluronic acid into a methacrylamide functionalized gelatin hydrogel, yielding the capacity to orthogonally manipulate hydrogel stiffness and HA content within the hydrogel. In addition to providing a platform to explore the effect of matriximmobilized HA on GBM cell expansion, invasion, and response to therapeutic inhibitors (Chen et al., 2017; Pedron et al., 2017a), we recently demonstrated it was possible to incorporate vascular cells into the hydrogel, yielding complex vascular networks that remodeled over time (Ngo and Harley, 2017). While 3D organoid-type cultures have fostered the production of "human brain-like tissue" (Sasai, 2013; Lancaster and Knoblich, 2014), so far attention has focused primarily on forebrain cell groups (Birey et al., 2017; Quadrato et al., 2017) or traumatic brain injury (Tang-Schomer et al., 2014), yielding opportunities to explore.

This project set out to examine the translation of a HA-modified gelatin hydrogel for the in vitro culture of NGC neurons. To date, these neural cells have not been studied ex vivo, and given their relevance in linking brain development to behavior, a permissive culture system will allow neuroscientists systematically to sort out the separate contributions of extracellular matrix components and growth factors to their survival, growth and development. Here we report examination of the ability to grow NGC neurons in vitro in such hydrogels without the assistance of growth factors.

\section{MATERIALS AND METHODS}

\section{Hydrogel Preparation and Characterization}

Gelatin methacrylamide (GelMA) and HA methacrylate (HAMA) were synthesized as described previously (Pedron et al., 2015). The degree of functionalization (\%DOF) was established by NMR as the ratio of methacrylamide groups $(5.5 \mathrm{ppm})$ to aromatic groups (7.3 ppm). Freeze dried GelMA (3.5 wt\%) was mixed with HAMA (0.5 wt \%) into PBS containing $0.02 \%(\mathrm{w} / \mathrm{v})$ lithium phenyl-2,4,6-trimethyl benzoyl phosphinate (LAP) as a photoinitiator until fully dissolved. Prepolymer solution was pipetted into Teflon molds $1.5 \mathrm{~mm}$ deep and $5 \mathrm{~mm}$ in diameter, and exposed to $10 \mathrm{~mW} / \mathrm{cm}^{2}$ UV light ( $365 \mathrm{~nm}$, LED AccuCure Spot) for $20 \mathrm{~s}$.

Mechanical analyses were performed on fully swollen disks as described previously (Pedron et al., 2013). Specimens were tested in compression at room temperature via an Instron 5493 (100 N load cell) mechanical testing apparatus ( $20 \%$ strain $/ \mathrm{min})$. The compressive modulus was determined from the linear region corresponding to $\sim 0-5 \%$ strain. Full laminin protein was replaced by CYIGSR sequence (generated at the Rockefeller University Proteomics Resource Center) previously described in the literature to support neuronal adhesion and growth (Mckinnon et al., 2013).

\section{Experimental Animals}

C57BL/6/J mice (Jackson Laboratories) were maintained on a $12 / 12$ light dark cycle with lights on at 7 a.m. and free access to water and food. Adult male and female animals were paired in the late afternoon to obtain timed pregnant dams and the morning when the formation of a vaginal plug was observed was counted as embryonic day 0 . All experimental procedures were performed in accordance with the NIH guide for care and use of animals and approved by the Animal Care and Use Committee 
at The Rockefeller University. Pregnant females at 12 days post conception (E12) were sacrificed by cervical dislocation. After cleaning the abdomen with an excess of $70 \%$ ethanol, a V-shape cut was performed onto the abdomen through the skin and muscle layers to expose the peritoneum. The uterine horns were removed and transferred into a clean plastic dish containing icecold dissection buffer (DB) consisting of Hanks balanced salt solution, HBSS, $\mathrm{Ca}^{2+}, \mathrm{Mg}^{2+}$ free (Gibco), $20 \mathrm{mM}$ D-Glucose (Sigma Aldrich) and 1\% penicillin/streptomycin (Gibco). The average number of embryos per pregnant female was 6-8.

\section{Hindbrain Dissection and Primary E12 Hindbrain Cell Cultures}

Under a dissecting stereomicroscope (Zeiss, model 10298), each embryo sac was separated from the intact uterine horns. The embryos were transferred to a clean plastic dish containing fresh ice-cold DB. The embryonic hindbrains were dissected according to Fantin et al. (2013) and the hindbrain dissociations were performed immediately after (see below). On average, the timing of dissection was 5 min per hindbrain.

\section{Hindbrain Dissociation}

The collected hindbrains in DB were transferred to the tissue culture hood, and they were transferred to one of the wells of a 24-multiwell plate containing fresh DB. The dissection buffer was then replaced with $500 \mu \mathrm{l}$ of the pre-warmed enzymatic cell dissociation reagent (StemPro ${ }^{\circledR}$ Accutase, Thermo Fisher Scientific) and incubated at $37^{\circ} \mathrm{C}$ for $5 \mathrm{~min}$ with gentle shaking. The hindbrains were then mechanically dissociated pipetting up and down 12 times using a 1,000 $\mu \mathrm{l}$ tip and 12 more times using a $200 \mu \mathrm{l}$ tip. Great care was taken in avoiding the formation of air bubbles during this step to maximize cell viability. The overall enzymatic and mechanical hindbrain dissociation step never exceeded $7 \mathrm{~min}$. The enzymatic digestion was stopped by adding $3 \mathrm{ml}$ of standard medium containing Dulbecco's Modified Eagle's Medium, DMEM (Gibco) supplemented with $10 \%$ fetal bovine serum (Gibco) and $1 \%$ penicillin/streptomycin (Gibco). The resulting cell suspension was filtered through a tube fitted with a cell strainer snap cap (35 $\mu \mathrm{m}$ nylon mesh, Falcon), transfer to a $15 \mathrm{ml}$ Falcon tube and further diluted with standard media to a final volume of $10 \mathrm{ml}$. After gentle inversion, an aliquot of the suspension was mixed with an equal volume of trypan blue and the viable cell yield (cells $/ \mathrm{ml}$ ) was estimated using a Neubauer chamber. Immediately after, the volume of the suspension was adjusted with media to the desired cell concentration per gel and the samples were centrifuged at $200 \mathrm{~g}$ for $4 \mathrm{~min}$ at room temperature. After discarding the supernatant, the resulting cell pellet was immediately resuspended on the prepolymer hydrogel mixture (see section below).

\section{Encapsulation of Dissociated Embryonic Hindbrain Cells}

We examined a library of hydrogel prepolymer solutions that varied by the overall total polymer concentration (5 or $4 \mathrm{wt} \%$ ), the degree of methacrylamide functionalization of the gelatin macromer (DOF: 85, 60, or 55\%), the fraction of HAMA
(0.5 or 1 wt\%) added to the GelMA solution, or the amount of LAP photoinitiator $(0.05,0.02 \mathrm{wt} \%)$ used to polymerize the hydrogel (Table 1). To promote the attachment of the embryonic hindbrain dissociated cells, a subset of hydrogels were functionalized with $800 \mu \mathrm{g} / \mathrm{ml}$ of a laminin peptide mimic (CYIGSR). The hydrogel components were dissolved in warm $\left(45^{\circ} \mathrm{C}\right)$ neurobasal serum-free medium consisting of neurobasal medium, 2\% B27 Supplement, 1\% Glutamax, 1\% penicillin/streptomycin. In one experiment, the components were dissolved in phosphate buffer saline as a control. All reagents were purchased from Gibco. The solution was kept at $37^{\circ} \mathrm{C}$ until the cell pellet was ready to be resuspended. After gently mixing, either $30 \mu \mathrm{l}(10$ million cells per $\mathrm{ml})$ of the embryonic hindbrain cell suspension was pipetted onto Teflon molds anchored on top of a glass slide and secured with binder clips (Pedron et al., 2013, 2017a). Hydrogels were subsequently created: disks of $5 \mathrm{~mm}$ diameter; the height of the gels was $1.5 \mathrm{~mm}$. The cell-laden prepolymer suspension was photopolymerized by exposure to $10 \mathrm{~mW} / \mathrm{cm}^{2} \mathrm{UV}$ light $(365 \mathrm{~nm})$ for 20 or $30 \mathrm{~s}$ (Table 1). Neurobasal medium was added to the gels immediately after polymerization to prevent dehydration. Each cell-seeded hydrogel was then transferred to a well of a 24-multiwell plate (Corning) containing neurobasal media and incubated at $37^{\circ} \mathrm{C}, 5 \% \mathrm{CO}_{2}$ on top of the plate of an orbital shaker (lowest speed setting) inside a cell culture incubator. Neurobasal medium was exchanged every 3 days over the course of all experiments.

\section{Live/Dead Cell Viability Assay}

The evaluation of cell viability was performed at 7 and 14 days in vitro (DIV) using the Live/Dead assay (Molecular Probes). After rinsing hydrogels with PBS (3X for $5 \mathrm{~min}$ each) they were incubated at room temperature with PBS containing $2 \mu \mathrm{M}$ Calcein-AM (stains the cytoplasm of viable cells green) and $4 \mu \mathrm{M}$ ethidium homodimer-1 (stains the nuclei of dead cells red). After $40 \mathrm{~min}$, the cell-laden hydrogels were rinsed in PBS and immediately imaged using a confocal microscope (Zeiss LSM 880 inverted confocal laser scanning microscope). Because the hydrogel area exceeded an individual confocal image field we acquired the complete hydrogel image using the tile module

TABLE 1 | The composition (overall hydrogel wt \%; GelMA/HAMA ratio; degree of methacrylamide functionalization of the gelatin macromer; inclusion of CYIGSR peptide), fabrication conditions (photoinitiator wt\%; UV exposure time), and resulting elastic modulus ( $n=4$ ) of the family of hydrogel samples used in the encapsulation of NGC cells.

\begin{tabular}{lllllll}
\hline $\begin{array}{l}\text { Total } \\
\text { wt } \%\end{array}$ & $\begin{array}{l}\text { GeIMA/HAMA } \\
\% \text { wt }\end{array}$ & $\begin{array}{l}\text { DOF } \\
\%\end{array}$ & $\begin{array}{l}\text { CYIGSR } \\
\boldsymbol{\mu} \mathbf{g} / \mathbf{m l}\end{array}$ & $\begin{array}{l}\mathbf{P I} \\
\mathbf{w t} \%\end{array}$ & $\begin{array}{l}\text { Time } \\
\mathbf{( s )}\end{array}$ & $\begin{array}{l}\text { Modulus } \\
\mathbf{k P a}\end{array}$ \\
\hline 5 & $4.5 / 0.5$ & 85 & 0 & 0.05 & 30 & $8.7 \pm 0.8$ \\
4 & $3 / 1$ & 60 & 0 & 0.02 & 20 & $5.0 \pm 0.3$ \\
4 & $3 / 1$ & 55 & 0 & 0.02 & 20 & $5.0 \pm 0.3$ \\
4 & $3 / 1$ & 55 & 800 & 0.02 & 20 & \\
4 & $3.5 / 0.5$ & 55 & 0 & 0.02 & 20 & $1.1 \pm 0.2$ \\
4 & $3.5 / 0.5$ & 55 & 800 & 0.02 & 20 &
\end{tabular}


included in the Zen confocal software (Zeiss). Starting at the surface and imaging down toward the center of the hydrogel, the first and last $\mathrm{z}$ positions for each $\mathrm{z}$-stack/tile were defined for a total thickness of 100 or $200 \mu \mathrm{m}$. The acquired image tiles were then combined into one final output by a stitching process using a $10 \%$ tile overlap. For automated cell counts, and estimation of cell viability, the stitched output images were imported to the $3 \mathrm{D}$ rendering software Imaris (Bitplane). We used the spot detection module, and spots were defined using cell count parameters for size and fluorescence strength of voxels to represent each cell soma. Parameters were then subjected to multiple image tests between manually counted and automated cell counts in multiple regions of interest of the imported tiled images to ensure accuracy. Because the quantification of spots using 200 and $100 \mu \mathrm{m}$ thick tiled z-stacks did not differ statistically within hydrogels, we did the analysis using the thinner tiled Z-stacks images. After validation, parameters were saved and then applied through the entire $100 \mu \mathrm{m} \mathrm{z}$-stacks and overall cell count data was obtained for each image. Cell viability was calculated as (number of green stained cells/number of total cells) X 100\%.

\section{Immunocytochemistry}

After 7 days in vitro, cell-laden hydrogels were rinsed $3 \mathrm{X}$ in PBS for $5 \mathrm{~min}$ per rinse and then fixed in 4\% paraformaldehyde (Sigma Aldrich) for $5 \mathrm{~min}$ at room temperature. After rinsing with PBS, the gels were permeabilized with $0.5 \%$ Triton X-100 (Sigma Aldrich), blocked with 5\% donkey serum (Invitrogen) and incubated overnight at $4^{\circ} \mathrm{C}$ with anti-TUJ1 mouse IgG primary antibody $(1: 1,000$, Biolegend). Hydrogels were rinsed in PBS and then incubated for $2 \mathrm{~h}$ at room temperature with donkey anti-mouse IgG secondary antibody conjugated to Alexa Fluor 488 (Life Technologies, 1:1,000). Cellular nuclei were counter stained with $0.4 \mu \mathrm{l} / \mathrm{ml}$ DAPI in PBS for $5 \mathrm{~min}$. The labeled hydrogels were examined and imaged using a Zeiss LSM 880 inverted confocal laser scanning microscope.

\section{Statistics}

Statistical analysis was performed via one-way analysis of variance (ANOVA) tests after which a Tukey-HSD post-hoc test was used. Analysis of hydrogel mechanical properties used $n=$ 5 constructs per group. Significance was set at $p<0.05$. Error bars are reported as standard error of the mean unless otherwise noted.

\section{RESULTS}

\section{Gelatin-Based Hydrogel Fabrication}

Both the biomechanical and cell-adhesive properties of $3 \mathrm{D}$ hydrogel matrices are critical for the viability and behavior of encapsulated cultured cells. Initially, the conditions used for the encapsulation of E12.5 hindbrain cells comprised the use of $5 \mathrm{wt} \%$ total polymer concentration with an $85 \%$ degree of functionalization and $0.05 \mathrm{wt} \%$ photoinitiator. These conditions resulted in highly crosslinked polymer network that led to stiff hydrogels $(8.7 \pm 0.8 \mathrm{kPa})$ and proved to be highly detrimental to the survival and differentiation of the embryonic cells (data not shown). With the aim of designing a more compliant hydrogel, in subsequent experiments, we resorted to a hydrogel containing a lower total polymer concentration (4 wt\%), lower degrees of functionalization of GelMA (60 or $55 \%)$, and photoinitiator concentration of $0.02 \mathrm{wt} \%$. The more permissive conditions $(1.1 \pm 0.2 \mathrm{kPa})$ for cell viability resulted from platforms containing 55\% DOF GelMA and $0.02 \mathrm{wt} \%$ concentration of photoinitiator. Interestingly, the addition of matrix immobilized HA to the GelMA hydrogel proved to be beneficial to sustain cell viability and differentiation, in some cases, of neuritic processes. The hydrogel composition employed
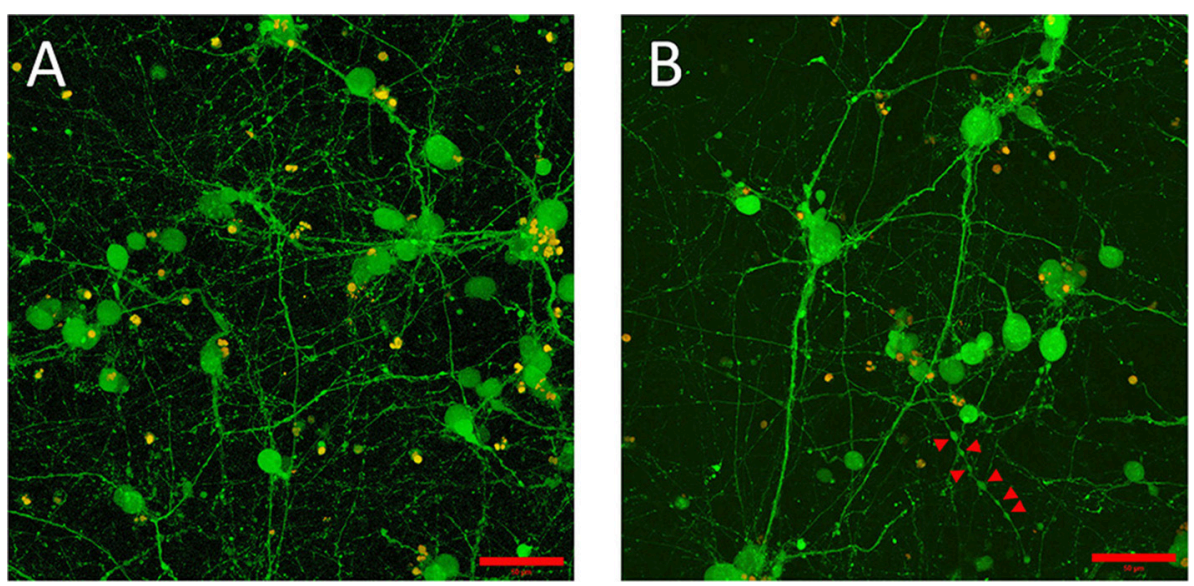

FIGURE 1 | Embryonic 12.5 (E12.5) hindbrain cells encapsulated in 3D hydrogel scaffolds (GelMA/HAMA: 3.5/0.5 wt\%, no laminin added) remained viable after 10 days in vitro (10DIV). (A) Maximum intensity projection depicts cell bodies projecting extensive processes adopting elaborated arborization patterns. Viable cells, shown in green, were labeled with calcein-AM (see Materials and Methods section for details). Scale bar: $50 \mu \mathrm{m}$. (B) Some encapsulated E12.5 hindbrain cells [same conditions as (A)] showed varicose neurites. Red arrows indicate the putative boutons along the neurite outgrowth. Scale bar: $50 \mu \mathrm{m}$. 
in the present report consisted of GelMA/HAMA 3.5/0.5\% and $0.02 \%$ photoinitiator.

\section{Survival and Growth of Nucleus Gigantocellularis (NGC) Neurons}

The initial, pre-gel viability of these NGC neurons measured by dye exclusion was in the range of $60-85 \%$. Cells encapsulated within the GelMA/HAMA (3.5/0.5 wt\%) hydrogels and their associated neurite processes remained viable for up to 2 weeks in vitro. Cell viability at the termination of experiments was found to be between 30 and $60 \%$ as measured by the Live/Dead assay. Viable neurons usually sent out neurite processes, in some cases with the primary neurite branching and producing second-level or even third-level neurite segments (Figure 1A). This phenomenon was highly variable, both within and between gels. In some cases, neurites displayed ovoid varicosities along their length, with complex neuronal processes observed after 2 weeks in vitro (Figure 1B). Importantly, neurons were distributed three-dimensionally throughout the hydrogel, with Figure 2 depicting NGC cells extending processes (color coded by depth of penetration) into the hydrogel scaffolding.

Experiments did not include a systematic comparison of results with and without laminin. Instead, it must be said that, while it was possible to see robust neuronal network formation in hydrogels containing the laminin peptide $(800 \mu \mathrm{g} / \mathrm{ml})$ (Figure 3), morphologically similar results could be observed in the absence of laminin (Figure 4).

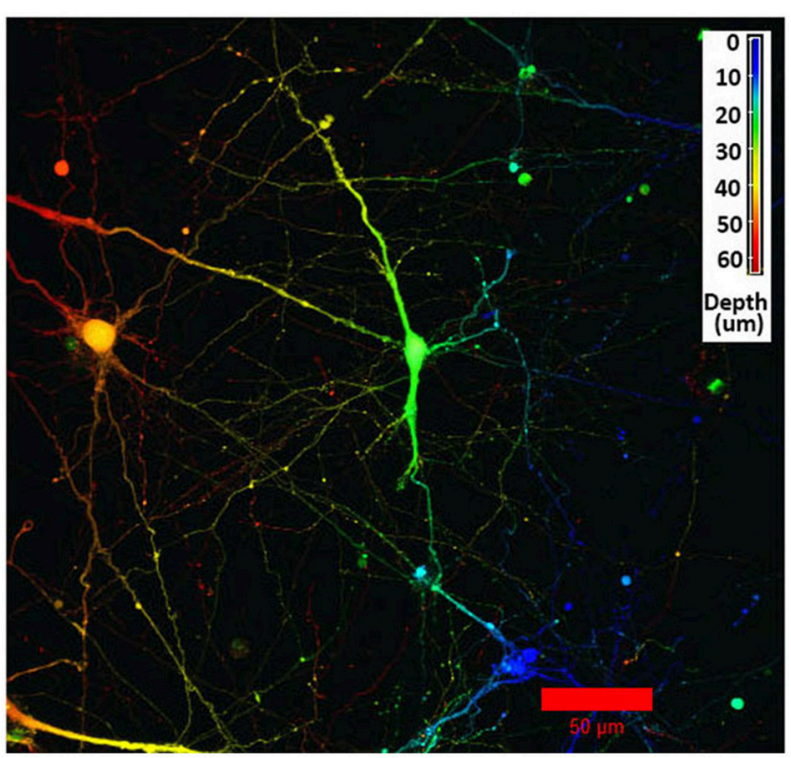

FIGURE 2 | Confocal microscopy image using depth decoding of an E12.5 hindbrain cell hydrogel culture (GelMA/HAMA: 3.5/0.5 wt\%, no laminin added) after 15DIV. Color decoding for the depth of the cells in the hydrogel along the $z$-axis is given. The difference in colors indicate the different planes along the z-axes. Scale bar: $50 \mu \mathrm{m}$

\section{Neuronal Differentiation of NGC Cells}

We subsequently explored the initial stages of neuronal differentiation of NGC cells maintained within the HA-modified

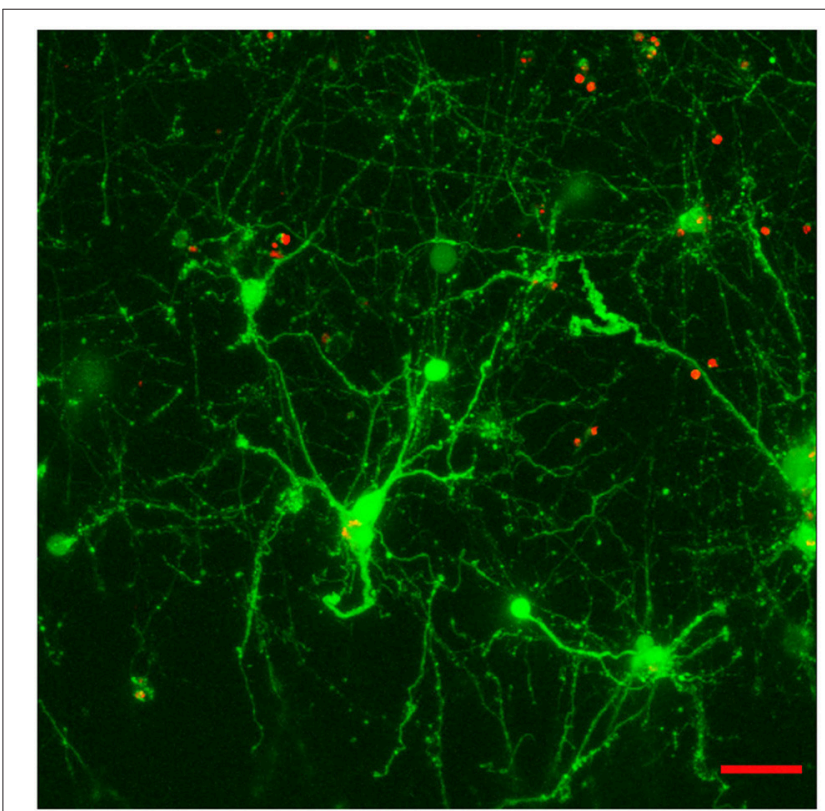

FIGURE 3 | Effect of the incorporation of a cell-adhesive laminin peptide mimic into E12.5 hindbrain cell-laden hydrogels (GelMA/HAMA: 3.5/0.5 wt\% plus $800 \mu \mathrm{g} / \mathrm{ml}$ CYIGSR) after 15DIV. Maximum projection of a $70 \mu \mathrm{m}$ z-stack showing that encapsulated cells mature and give out neurites in the presence of laminin mimic. Scale bar: $50 \mu \mathrm{m}$

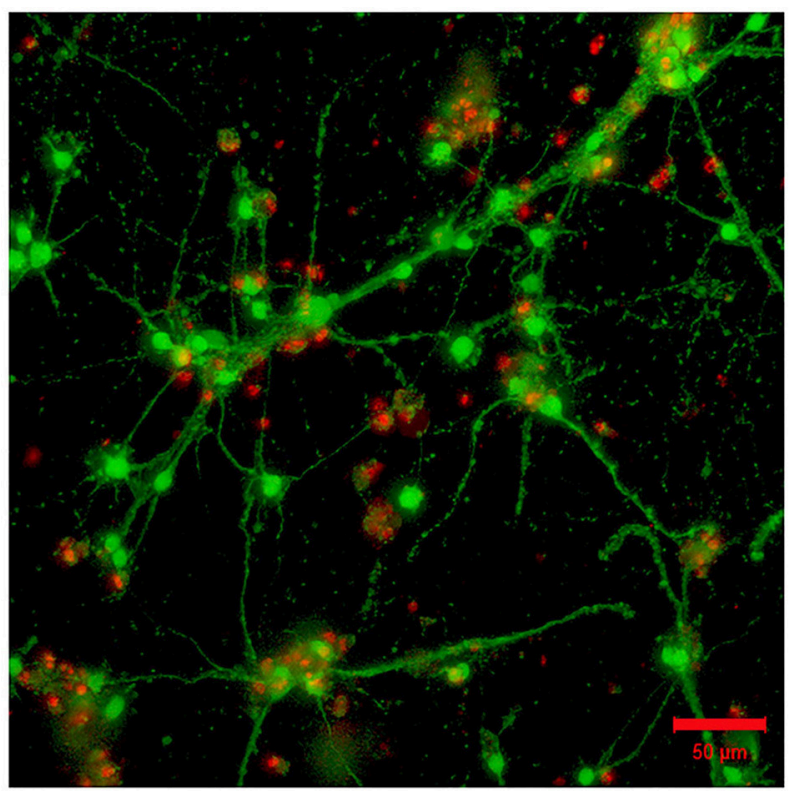

FIGURE 4 | Absence of laminin in the 3D hydrogel (GelMA/HAMA: 3.5/0.5 $w t \%)$ is permissive for the outgrowth of neurites from encapsulated embryonic hindbrain cells after 15DIV. Scale bar: $50 \mu \mathrm{m}$. 
GelMA hydrogel. Excitingly, viable NGC cells adopted a neuronal morphology. Immunocytochemistry using an antibody against neuron-specific class III-beta-tubulin, a microtubule component used as a neuronal marker, confirmed that the cells encapsulated within the GelMA hydrogels displayed a neuronal phenotype (Figure 5). Further, after 7 days of culture in the hydrogels in vitro, cellular processes exhibited morphologies suggesting that they could form more complex neuronal networks.

\section{DISCUSSION}

This work shows that it is possible to grow embryonic reticular formation neurons in $3 \mathrm{D}$ hydrogels in the absence of growth factors or nerve cell adhesion molecules. The neurons used, from the medial medullary reticular formation, which would include the large cells of nucleus gigantocellularis, are crucial for CNS arousal and the initiation of a wide range of behaviors. It remains to be seen whether optimum conditions for such neurons differ from those, for example, in the forebrain (Birey et al., 2017).

We selected gelatin-HA hydrogel as the base material for the fabrication of culture platforms for NGC neurons due to their success in hosting mammalian cells (Du et al., 2017), and our previous experience with cancerous glial cells (Chen et al., 2017; Pedron et al., 2017a,b). Biophysical properties of gelatin hydrogels can be easily manipulated by dialing concentration, degree of functionalization and photoreaction conditions (Pedron and Harley, 2013; Pedron et al., 2013, 2015, 2017a). Primary neural cells are very vulnerable to in vitro culture

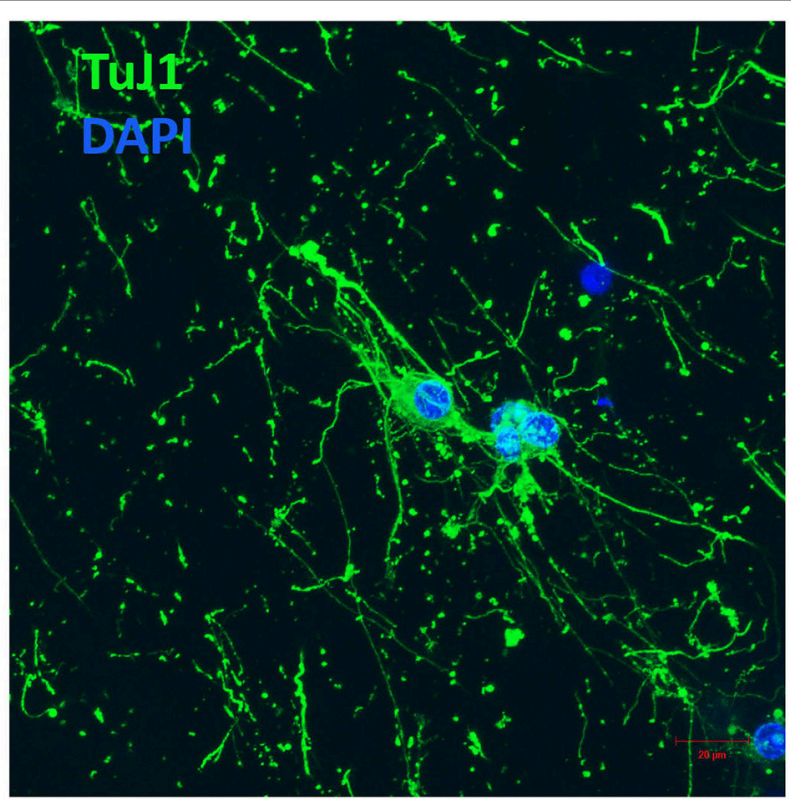

FIGURE 5 | E12 hindbrain cells encapsulated in GelMA/HAMA: 3.5/0.5 wt\% plus $800 \mu \mathrm{g} / \mathrm{ml}$ CYIGSR display a neuronal phenotype after 7DIV. Hydrogels were immunostained with Tuj-1, an antibody against neuron-specific class III-beta tubulin (see Materials and Methods section for details). Scale bar: $20 \mu \mathrm{m}$. conditions and need very specific environmental conditions (e.g., mechanical stress, porosity, degradability, attachment sites) to keep them viable and functional. Very mild conditions of polymerization, low polymer concentration, and compliant resulting gels (Palazzolo et al., 2015) are known to be necessary for achieving a high initial cell viability, very important in the case of neurons that do not proliferate. In this case, the most permissive gels presented an elastic modulus around $1.1 \mathrm{kPa}$, and cells show long interconnected processes with the appearance of varicosities (Figure 1). In photo-initiated reactions, for instance, time, and intensity of exposure to UV light is a critical variable that needs to be considered during cell encapsulation that also engages material mechanical compliance. Ongoing work focuses on developing more compliant hydrogel constructs to ensure greater and more stable neuronal outgrowth. Indeed, matrix architecture is highly important in $3 \mathrm{D}$ microenvironments to allow for neurite extension at the same time as nutrients and oxygen flow. Current advancements in formation of endothelial networks within these constructs (Chen et al., 2017) could demonstrate as a prospective solution for the alteration of oxygen and biomolecule transport within the hydrogel network. Future directions aim to achieve a broader array of matrix architectures in order to optimize cell culture conditions: alternative crosslinking chemistries and simplification of sample fabrication by using spatially graded hydrogels (Pedron et al., 2017b) using microfluidics.

Despite our success in growing these neurons that are crucial for the initiation of behavior, several aspects of the work indicated that this methodology deserves further development. First, our $3 \mathrm{D}$ gels should in principle be amenable for electrophysiological recording. We note that it is harder to visualize the surfaces of target neurons through the gel than in a simple bath; and with the micropipette entering the gel from the right, advancing in the gel toward the target neuron pushes the gel (and the neuron) to the left even to the point where the neuron would be deformed. Second, despite exquisite attention to methodological detail, we noted variability in neuron viability and the consistency of neuron processes. In particular, we observed in some cases neurites presenting non uniform widths suggestive of varicosities or putative boutons. Although signs of axonal degeneration cannot be ruled out, future studies should address the latter possibility exploring the presence of vesicle associated proteins such as synapsin (Neto et al., 2014). In trying to explain the variability in neuron viability, one possibility lies with the method of neuronal concentration in the pellet of tissue immediately before adding the pre-polymer solution. Another possible cause of variability could stem from the crosslinking reaction and the hydrogel network formed thereof. Moreover, although adding laminin-derived adhesion peptide to the gel preparation did not systematically improve results, we had a small number of experiments in which the presence of the peptide mimic did improve viability and neuronal network formation, opening the door to exploring alternative methods to present adhesion moieties to NGC cells. One possible strategy to explore a wider range of combinations of adhesion molecules or a wider range of activity inducing factors would be the use of microfluidic patterning tools that we have previously described to create 
gelatin hydrogels containing embossed patterns of biomolecules (Pedron et al., 2013; Mahadik et al., 2015). Such tools can build on the observations here to test a wider range of concentrations, create patterns employing either or both soluble vs. hydrogelimmobilized signals, and would be ideally suited to examine a wider range of bioactive factors that might enhance neuron outgrowth that have been previously identified in the literature such as EGF and bFGF (Rao, 1999; Maric et al., 2003).

\section{CONCLUSION}

For the first time, we report the successful in vitro culture and growth of primary NGC neurons, crucial for the initiation of behavior, within gelatin-based biomaterial platforms. HA-functionalized GelMA hydrogels, previously identified for the growth and expansion of glioblastoma cells, provides a 3D-hydrogel environment in which NGC viability, neuronal process extension, and the expression of markers of neuronal differentiation could be observed. Ongoing efforts are exploring modifications to the gelatin-based hydrogel biophysical properties to achieve a robust growth and stable neural differentiation for prolonged periods of time.

\section{REFERENCES}

Alessandri, K., Feyeux, M., Gurchenkov, B., Delgado, C., Trushko, A., Krause, K. H., et al. (2016). A 3D printed microfluidic device for production of functionalized hydrogel microcapsules for culture and differentiation of human Neuronal Stem Cells (hNSC). Lab Chip 16, 1593-1604. doi: 10.1039/C6LC00133E

Baier, C., Baader, S. L., Jankowski, J., Gieselmann, V., Schilling, K., Rauch, U., et al. (2007). Hyaluronan is organized into fiber-like structures along migratory pathways in the developing mouse cerebellum. Matrix Biol. 26, 348-358. doi: 10.1016/j.matbio.2007.02.002

Birey, F., Andersen, J., Makinson, C. D., Islam, S., Wei, W., Huber, N., et al. (2017). Assembly of functionally integrated human forebrain spheroids. Nature 545, 54-59. doi: 10.1038/nature22330

Caballero, D., Kaushik, S., Correlo, V. M., Oliveira, J. M., Reis, R. L., and Kundu, S. C. (2017). Organ-on-chip models of cancer metastasis for future personalized medicine: from chip to the patient. Biomaterials 149, 98-115. doi: 10.1016/j.biomaterials.2017.10.005

Calderon, D. P., Kilinc, M., Maritan, A., Banavar, J. R., and Pfaff, D. (2016). Generalized CNS arousal: an elementary force within the vertebrate nervous system. Neurosci. Biobehav. Rev. 68, 167-176. doi: 10.1016/j.neubiorev.2016.05.014

Chen, J. W. E., Pedron, S., and Harley, B. A. C. (2017). The combined influence of hydrogel stiffness and matrix-bound hyaluronic acid content on glioblastoma invasion. Macromol. Biosci. 17:1700018-n/a. doi: 10.1002/mabi.2017 00018

Chung, B., Esmaeili, A. A., Gopalakrishna-Pillai, S., Murad, J. P., Andersen, E. S., Kumar Reddy, N., et al. (2017). Human brain metastatic stroma attracts breast cancer cells via chemokines CXCL16 and CXCL12. npj Breast Cancer 3:6. doi: 10.1038/s41523-017-0008-8

Di Lullo, E., and Kriegstein, A. R. (2017). The use of brain organoids to investigate neural development and disease. Nat. Rev. Neurosci. 18:573. doi: $10.1038 / \mathrm{nrn} .2017 .107$

Du, C., Collins, W., Cantley, W., Sood, D., and Kaplan, D. L. (2017). Tutorials for electrophysiological recordings in neuronal tissue engineering. ACS Biomater. Sci. Eng. 3, 2235-2246. doi: 10.1021/acsbiomaterials.7b00318

\section{AUTHOR CONTRIBUTIONS}

AM and SP designed experiments, modified and adapted $2 \mathrm{D}$ cell culture for $3 \mathrm{D}$ hydrogel cultures, performed results interpretation and wrote the manuscript. AM and MC performed data analysis. $\mathrm{MK}$ and IT worked out conditions for $2 \mathrm{D}$ embryonic hindbrain primary cell cultures, $\mathrm{BH}$ and $\mathrm{DP}$ designed experiments, performed results interpretation, and manuscript writing.

\section{ACKNOWLEDGMENTS}

Research reported in this publication was supported by the National Cancer Institute of the National Institutes of Health under Award Number R01 CA197488. The content is solely the responsibility of the authors and does not necessarily represent the official views of the NIH. This material is based upon work supported by the National Science Foundation under Grant No. $1254738(\mathrm{BH})$. The authors are also grateful for additional funding provided by the New York Neuroscience Foundation (DP), the Department of Chemical \& Biomolecular Engineering and the Carl R. Woese Institute for Genomic Biology at the University of Illinois at Urbana-Champaign.

Fantin, A., Vieira, J. M., Plein, A., Maden, C. H., and Ruhrberg, C. (2013). The embryonic mouse hindbrain as a qualitative and quantitative model to study the molecular and cellular mechanisms of angiogenesis. Nat. Protoc. 8, 418-429. doi: 10.1038/nprot.2013.015

Gu, Q., Tomaskovic-Crook, E., Lozano, R., Chen, Y., Kapsa, R. M., Zhou, Q., et al. (2016). Functional 3D neural mini-tissues from printed gel-based bioink and human neural stem cells. Adv. Healthcare Mater. 5, 1429-1438. doi: 10.1002/adhm.201600095

Gurkan, U. A., Fan, Y., Xu, F., Erkmen, B., Urkac, E. S., Parlakgul, G., et al. (2013). Simple precision creation of digitally specified, spatially heterogeneous, engineered tissue architectures. Adv. Mater. 25, 1192-1198. doi: 10.1002/adma.201203261

Heffernan, J. M., Overstreet, D. J., Le, L. D., Vernon, B. L., and Sirianni, R. W. (2015). Bioengineered scaffolds for 3D analysis of glioblastoma proliferation and invasion. Ann. Biomed. Eng. 43, 1965-1977. doi: 10.1007/s10439-014-1223-1

Jones, B. E., and Yang, T. Z. (1985). The efferent projections from the reticular formation and the locus coeruleus studied by anterograde and retrograde axonal transport in the rat. J. Comp. Neurol. 242, 56-92. doi: 10.1002/cne.902420105

Kingsmore, K. M., Logsdon, D. K., Floyd, D. H., Peirce, S. M., Purow, B. W., and Munson, J. M. (2016). Interstitial flow differentially increases patient-derived glioblastoma stem cell invasion via CXCR4, CXCL12, and CD44-mediated mechanisms. Integr. Biol. 8, 1246-1260. doi: 10.1039/C6IB00167J

Lampe, K. J., Antaris, A. L., and Heilshorn, S. C. (2013). Design of threedimensional engineered protein hydrogels for tailored control of neurite growth. Acta Biomater. 9, 5590-5599. doi: 10.1016/j.actbio.2012.10.033

Lancaster, M. A., and Knoblich, J. A. (2014). Organogenesis in a dish: modeling development and disease using organoid technologies. Science 345:1247125. doi: $10.1126 /$ science. 1247125

Liu, X., Pfaff, D. W., Calderon, D. P., Tabansky, I., Wang, X., Wang, Y., et al. (2016). Development of electrophysiological properties of nucleus gigantocellularis neurons correlated with increased CNS arousal. Dev. Neurosci. 38, 295-310. doi: 10.1159/000449035

Mahadik, B. P., Pedron Haba, S., Skertich, L. J., and Harley, B. A. (2015). The use of covalently immobilized stem cell factor to selectively affect 
hematopoietic stem cell activity within a gelatin hydrogel. Biomaterials 67, 297-307. doi: 10.1016/j.biomaterials.2015.07.042

Maric, D., Maric, I., Chang, Y. H., and Barker, J. L. (2003). Prospective cell sorting of embryonic rat neural stem cells and neuronal and glial progenitors reveals selective effects of basic fibroblast growth factor and epidermal growth factor on self-renewal and differentiation. J. Neurosci. 23:240. doi: 10.1523/JNEUROSCI.23-0100240.2003

Martin, E. M., Devidze, N., Shelley, D. N., Westberg, L., Fontaine, C., and Pfaff, D. W. (2011). Molecular and neuroanatomical characterization of single neurons in the mouse medullary gigantocellular reticular nucleus. J. Comp. Neurol. 519, 2574-2593. doi: 10.1002/ cne. 22639

Martin, E. M., Pavlides, C., and Pfaff, D. (2010). Multimodal sensory responses of nucleus reticularis gigantocellularis and the responses\&\#039; relation to cortical and motor activation. J. Neurophysiol. 103:2326. doi: $10.1152 /$ jn.01122.2009

Mckinnon, D. D., Kloxin, A. M., and Anseth, K. S. (2013). Synthetic hydrogel platform for three-dimensional culture of embryonic stem cell-derived motor neurons. Biomater. Sci. 1, 460-469. doi: 10.1039/c3bm00166k

Neto, E., Alves, C. J., Sousa, D. M., Alencastre, I. S., Lourenço, A. H., Leitão, L., et al. (2014). Sensory neurons and osteoblasts: close partners in a microfluidic platform. Integr. Biol. 6, 586-595. doi: 10.1039/C4IB00035H

Neves, L. S., Rodrigues, M. T., Reis, R. L., and Gomes, M. E. (2016). Current approaches and future perspectives on strategies for the development of personalized tissue engineering therapies. Expert Rev. Precision Med. Drug Dev. 1, 93-108. doi: 10.1080/23808993.2016.1140004

Ngo, M. T., and Harley, B. A. (2017). The influence of hyaluronic acid and glioblastoma cell coculture on the formation of endothelial cell networks in gelatin hydrogels. Adv. Healthcare Mater. 6:1700687-n/a. doi: 10.1002/adhm.201700687

Palazzolo, G., Broguiere, N., Cenciarelli, O., Dermutz, H., and ZenobiWong, M. (2015). Ultrasoft alginate hydrogels support long-term threedimensional functional neuronal networks. Tissue Eng. Part A 21, 2177-2185. doi: 10.1089/ten.tea.2014.0518

Pedron, S., and Harley, B., A. (2013). Impact of the biophysical features of a 3D gelatin microenvironment on glioblastoma malignancy. J. Biomed. Mater. Res. Part A 101, 3404-3415. doi: 10.1002/jbm.a.34637

Pedron, S., Becka, E., and Harley, B. A. (2015). Spatially gradated hydrogel platform as a 3D engineered tumor microenvironment. Adv. Mater. 27, 1567-1572. doi: 10.1002/adma.201404896

Pedron, S., Becka, E., and Harley, B. A.(2013). Regulation of glioma cell phenotype in 3D matrices by hyaluronic acid. Biomaterials 34, 7408-7417. doi: 10.1016/j.biomaterials.2013.06.024

Pedron, S., Hanselman, J. S., Schroeder, M. A., Sarkaria, J. N., and Harley, B. A. C. (2017a). Extracellular hyaluronic acid influences the efficacy of egfr tyrosine kinase inhibitors in a biomaterial model of glioblastoma. Adv. Healthcare Mater. 6:1700529. doi: 10.1002/adhm.201700529
Pedron, S., Polishetty, H., Pritchard, A. M., Mahadik, B. P., Sarkaria, J. N., and Harley, B. A. C. (2017b). Spatially graded hydrogels for preclinical testing of glioblastoma anticancer therapeutics. MRS Commun. 7, 442-449. doi: $10.1557 / \mathrm{mrc} .2017 .85$

Pfaff, D. W. (2006). Brain Arousal and Information Theory: Neural and Genetic Mechanisms. Cambridge, MA: Harvard University Press.

Pfaff, D. W., Martin, E. M., and Faber, D. (2014). Origins of arousal: roles for medullary reticular neurons. Trends Neurosci. 35, 468-476. doi: 10.1016/j.tins.2012.04.008

Pradhan, S., Hassani, I., Clary, J. M., and Lipke, E. A. (2016). Polymeric biomaterials for in vitro cancer tissue engineering and drug testing applications. Tissue Eng. Part B Rev. 22, 470-484. doi: 10.1089/ten.teb.2015.0567

Quadrato, G., Nguyen, T., Macosko, E. Z., Sherwood, J. L., Min Yang, S., Berger, D. R., et al. (2017). Cell diversity and network dynamics in photosensitive human brain organoids. Nature 545, 48-53. doi: 10.1038/nature22047

Rao, M. S. (1999). Multipotent and restricted precursors in the central nervous system. Anatomical Record 257, 137-148.

Rape, A., Ananthanarayanan, B., and Kumar, S. (2014). Engineering strategies to mimic the glioblastoma microenvironment. Adv. Drug Delivery Rev. 79-80, 172-183. doi: 10.1016/j.addr.2014.08.012

Sasai, Y. (2013). Cytosystems dynamics in self-organization of tissue architecture. Nature 493, 318-326. doi: 10.1038/nature11859

Seidlits, S. K., Khaing, Z. Z., Petersen, R. R., Nickels, J. D., Vanscoy, J. E., Shear J. B., et al. (2010). The effects of hyaluronic acid hydrogels with tunable mechanical properties on neural progenitor cell differentiation. Biomaterials 31 , 3930-3940. doi: 10.1016/j.biomaterials.2010.01.125

Tang-Schomer, M. D., White, J. D., Tien, L. W., Schmitt, L. I., Valentin, T. M., Graziano, D. J., et al. (2014). Bioengineered functional brain-like cortical tissue. Proc. Natl. Acad. Sci. U. S. A. 111, 13811-13816. doi: 10.1073/pnas.1324214111

Wu, H. B., Stavarache, M., Pfaff, D. W., and Kow, L. M. (2007). Arousal of cerebral cortex electroencephalogram consequent to high-frequency stimulation of ventral medullary reticular formation. Proc. Natl. Acad. Sci. U. S. A. 104, 18292-18296. doi: 10.1073/pnas.0708620104

Xiao, W., Sohrabi, A., and Seidlits, S. K. (2017). Integrating the glioblastoma microenvironment into engineered experimental models. Future Sci. OA 3:FSO189. doi: 10.4155/fsoa-2016-0094

Conflict of Interest Statement: The authors declare that the research was conducted in the absence of any commercial or financial relationships that could be construed as a potential conflict of interest.

Copyright (๑) 2018 Magariños, Pedron, Creixell, Kilinc, Tabansky, Pfaff and Harley. This is an open-access article distributed under the terms of the Creative Commons Attribution License (CC BY). The use, distribution or reproduction in other forums is permitted, provided the original author(s) and the copyright owner are credited and that the original publication in this journal is cited, in accordance with accepted academic practice. No use, distribution or reproduction is permitted which does not comply with these terms. 